\title{
Pion and eta production in elementary and heavy-ion collisions at SIS energies
}

\author{
M. Gumberidze ${ }^{1, \star}$ for the HADES Collaboration \\ ${ }^{1}$ Technische Universität Darmstadt, 64289 Darmstadt, Germany
}

\begin{abstract}
A main emphasis of the HADES program is on the dielectron signal from the early phase of heavy-ion collisions. We discuss the data with respect to the emission of charged hadrons, specifically the production of $\pi^{+}$and $\pi^{-}$mesons, which are related to neutral pions representing a dominant contribution to the dielectron yield. In addition, inclusive pion production obtained with the photon-conversion method is disscused. Preliminary results from $\mathrm{Au}+\mathrm{Au}$ at $1.23 \mathrm{AGeV}$ are presented.
\end{abstract}

\section{Introduction}

Pion production is the dominating inelastic process in nucleus-nucleus collisions. At beam energies in the range of $1-2 \mathrm{GeV}$ per nucleon pions are predominantly created via the excitation and the decay of hadron resonances. The $\Delta(1232)$ baryon resonance is most copiously produced, but as the incident energy increases, higher-lying resonances, such as $\mathrm{N}^{*}(1440), \mathrm{N}^{*}(1520), \mathrm{N}^{*}(1535), \mathrm{N}^{*}(1600)$, etc., also contribute to pion production in a wide energy range and to production close to the threshold (pions contribute to the production of heavier mesons close to their respective thresholds). These contributions stand out clearly in the dielectron invariant mass distributions measured in elementary and heavy-ion collisions at SIS18 energies. A detailed description of the resonance and meson production in elementary hadronic collisions is indeed a crucial ingredient of transport model calculations used to 2 frvb describe particle production at these energies.

\section{The HADES experiment}

The High Acceptance DiElecton Spectrometer (HADES), installed at SIS18 at the GSI Helmholtzzentrum für Schwerionenforschung (Germany), is a unique apparatus to study dielectron $\left(\mathrm{e}^{+} \mathrm{e}^{-}\right)$and hadron production in heavy-ion collisions, as well as in proton- and pion-induced reactions in the energy range of $1-4 \mathrm{GeV}$. HADES is a fixed-target experiment with large angular acceptance $\left(18^{\circ}\right.$ $-85^{\circ}$ in polar and almost full coverage in azimuthal angles). The setup comprises a Ring Imaging Cerenkov Detector (RICH) in the field-free region around the target, a low-mass tracking system, a superconducting toroidal magnet, and additional detectors for particle identification. A detailed description of the setup can be found in [1]. As of now, HADES has measured rare and penetrating probes in $\mathrm{C}+\mathrm{C}, \mathrm{Ar}+\mathrm{KCl}, p+p, d+p, p+\mathrm{Nb}$, and $\mathrm{Au}+\mathrm{Au}$ collisions. Measurements in $p+p$ collisions

^e-mail: m.gumberidze@gsi.de 
at various beam energies $(1.25,2.2,3.5 \mathrm{GeV})$ and $d+p$ (at $1.25 \mathrm{GeV})$ provided valuable constraints on the various sources contributing to the dielectron signal and allowed to obtain model-independent reference spectra for studies of cold ( $p+\mathrm{A}$ collisions), and hot and dense matter effects $(\mathrm{A}+\mathrm{A}$ collisions).

\section{Charged pion reconstruction}

The interpretation of the dilepton spectra relies on a precise knowledge of the underlying hadronic cocktail composed of components from various sources contributing to the differential yields. The neutral mesons, and foremost the $\pi^{0}$ constitute the source of the bulk of the detected dielectron pairs, namely via the $\pi^{0}$ Dalitz and $\gamma \gamma$ decays. The number of $\pi^{0}$ produced in the reaction can be estimated as an average of $\pi^{+}$and $\pi^{-}$yields, due to isospin symmetry. It serves as an input for the normalization of the dielectron data obtained in the same experiment.

Identification of charged pions is done based on velocity of particles detected in the time-of-flight detectors as a function of momentum. Reconstructed yields of pions were corrected for efficiency and purity. Preliminary transverse-mass spectra of negatively charged pions in different intervals of rapidity are shown in Fig. 1. Experimental data (colored points) have been fitted in each rapidity bin using the sum of two exponential functions (colored lines) in order to extrapolate down to transverse mass 0 . Statistical errors are neglegible thanks to our large data sample (plotted spectra correspond to only $4 \%$ of the collected data !). Systematic errors are estimated to be of the order of $5 \%$.

Thanks to the wide acceptance of the present experiment we will be able to do a direct comparison of our $\mathrm{Au}+\mathrm{Au}$ at $1.23 \mathrm{AGeV}$ data with the results of previous experiments e.g. FOPI [2].
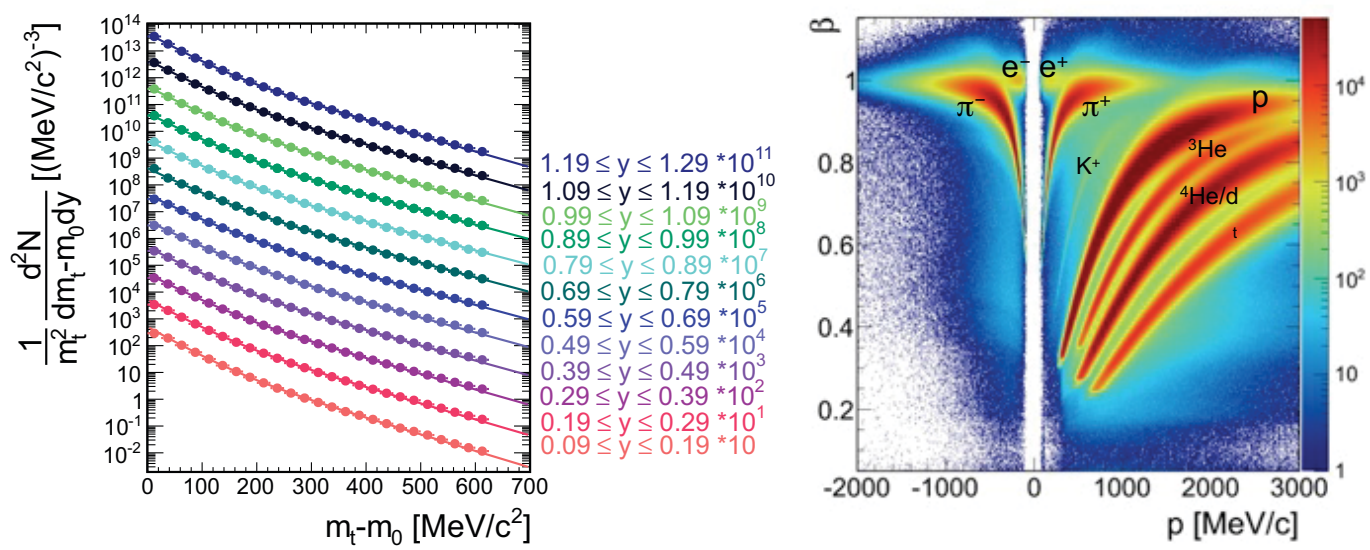

Figure 1. (Color online) Transverse-mass distributions for negatively charged pions in different slices of rapidity (left hand-side). Velocity vs. charge x momentum of charged particles as seen by the HADES detector (right hand-side).

\section{Full conversion method}

The contribution of the neutral mesons to the dilepton mass spectra is given by their Dalitz decays. However full reconstruction of the meson requires measuring also the $\gamma$ from Dalitz decays. Furthermore, $\pi^{0}$ and $\eta$ mesons decay dominantly into two photons, and this channel can be studied as 
well. As HADES is presently not equipped with an electromagnetic calorimeter direct measurement of photons is not possible. As an alternative, a $\gamma$-conversion technique in which meson decay photons are detected via their external conversion into an $e^{+} e^{-}$pair has been used (for details see [3]). In this case meson reconstruction is done by identifing lepton tracks and joining them into opposite-sign pairs $\left(e^{+} e^{-}\right)$, and by further combining those dilepton pairs into $e^{+} e^{-} e^{+} e^{-}$multiplets.

In order to suppress combinations of uncorrelated leptons, several topological cuts are applied on the opening angle between the leptons and the reconstructed pairs. Calculating the invariant mass $M_{e^{+} e^{-} e^{+} e^{-}}$and setting appropriate mass cuts allows to select the $\pi^{0}$ and $\eta$ mesons, respectively. Phase space coverage of $\pi^{0}$ as a function of transverse momentum and rapidity is shown in Fig. 2. As one can see, in rapidity we are covering the mid-rapidity region. The cutoff of the $\pi^{0}$ yield at $\mathrm{p}_{\perp}<0.2$ $\mathrm{GeV}_{c}$ is caused mostly by the strong bending of low-momentum tracks in the HADES magnetic field.

The resulting $M_{e^{+} e^{-} e^{+} e^{-}}$distribution from $\mathrm{Au}+\mathrm{Au}$ at $1.23 \mathrm{AGeV}$ is shown in Fig. 3. A prominent peak appears at the mass of $\pi^{0}$ meson on top of a combinatorial background, which can be well described by event-mixed continuum (see Fig. 3).
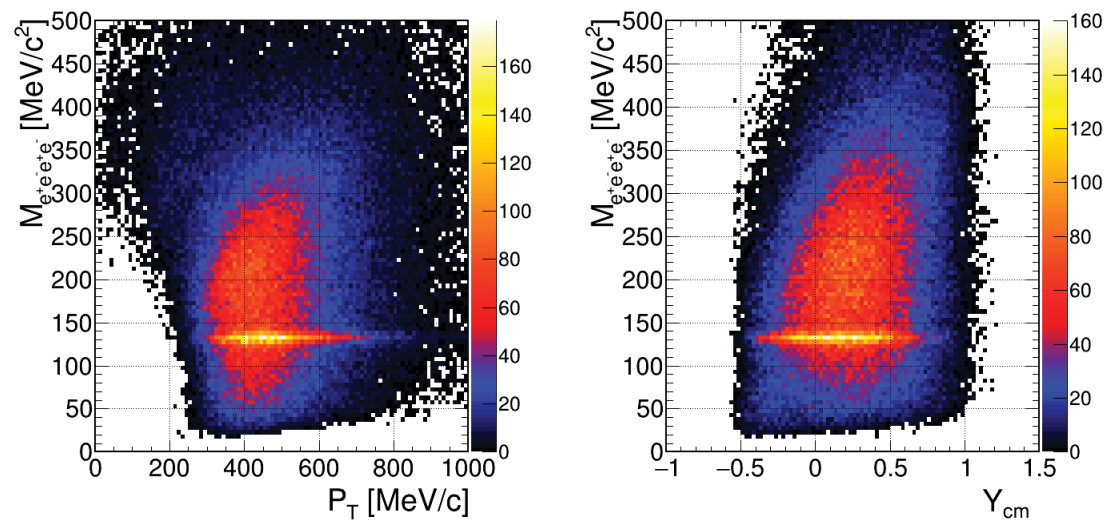

Figure 2. Phase space coverage of four-lepton $e^{+} e^{-} e^{+} e^{-}$multiplets.

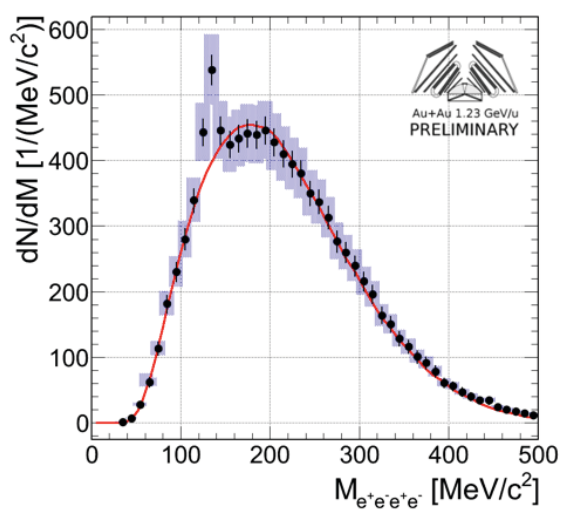

Figure 3. Invariant mass distribution of all $e^{+} e^{-} e^{+} e^{-}$multiplets measured with HADES in the $\mathrm{Au}+\mathrm{Au}$ at $1.23 \mathrm{AGeV}$ reaction. The background of uncorrelated combinations obtained with event mixing is shown as well (red points). 


\section{Summary}

HADES has measured charged pion yields in $\mathrm{Au}+\mathrm{Au}$ collisions at $1.23 \mathrm{AGeV}$ with unprecedented statistics. The external conversion method allowed to also measure neutral mesons, despite the lack of dedicated photon detector. This provides a necessary baseline for further analysis of the electromagnetic radiation originating from the hot and dense hadronic medium formed in these reactions. Furthermore, the high-quality charged pion data will allow to provide results on collective and correlation effects in the future.

In the future an electromagnetic calorimeter will be installed at HADES and it will largely improve the reconstruction of photons and meutral mesons.

This work has been supported by TU Darmstadt: VHNG823, Helmholtz Alliance HA216/EMMI and GSI.

\section{References}

[1] G. Agakishiev et al. (HADES Collaboration), Eur. Phys. J. A 41, 243 (2009)

[2] W. Reisdorf et al. (FOPI Collaboration) Nucl. Phys. A 781 459-508 (2007)

[3] G. Agakishiev et al. (HADES Collaboration), Phys. Rev. C 88, 024904 (2013) 